\title{
Should We Delay Urodynamic Study When Patients With Spinal Cord Injury Have Asymptomatic Pyuria?
}

\author{
EunYoung Kim, MD ${ }^{1}$, Hye Jin Lee, MD $^{1}$, Onyoo Kim, MD ${ }^{1}$, In Suk Park, RN² , Bum-Suk Lee, MD, MPH
}

${ }^{1}$ Department of Physical Medicine and Rehabilitation, National Rehabilitation Center, Seoul;

${ }^{2}$ Department of Nursing, National Rehabilitation Center, Seoul, Korea

Objective To assess the incidence of urinary tract infection (UTI) with post-urodynamic study (post-UDS) in patients with spinal cord injury (SCI) and study its relationship with pre-UDS pyuria.

Methods Patients with SCI who were hospitalized and underwent UDS during a 4-year period were reviewed. Patients with pre-test lower urinary tract symptoms were excluded. Urinalysis and urine culture were performed before and 24 hours after UDS. Prophylactic antibiotics were administered for 5 days starting from the morning of the UDS. UTI was defined as bacteriuria with accompanying symptoms.

Results Of 399 patients reviewed, 209 (52.4\%) had pyuria in pre-UDS urinalysis, and 257 (64.4\%) had bacteriuria in pre-UDS culture. Post-UDS UTI occurred in 6 (1.5\%) individuals who all complained of fever: 5 (2.4\%) of the post-UDS UTI cases occurred in patients with pre-UDS pyuria, and $1(0.5 \%)$ in a person without. The differences between groups were not statistically significant $(\mathrm{p}=0.218)$. Of 221 patients with bacteriuria (gram-negative isolates) on pre-UDS culture, resistance to ciprofloxacin, cephalosporin, and trimethoprim/sulfamethoxazole (TMP/SMT) was noted in $52.9 \%$ ( 117 cases), $57.0 \%$ ( 126 cases), and $38.9 \%$ ( 86 cases), respectively.

Conclusion No difference was found in the prevalence of post-UDS UTI based on the presence of pyuria in preUDS urinalysis. UDS may be performed even in SCI cases of pre-UDS pyuria without increasing the prevalence of post-UDS UTI if prophylactic antibiotics are administered. TMP/SMT could be used as a first-line antibiotic for the prevention of post-UDS UTI in Korea.

Keywords Pyuria, Bacteriuria, Urinalysis, Urodynamics, Spinal cord injuries, Urinary tract infections

Received November 18, 2020; Revised January 4, 2021; Accepted February 9, 2021; Published online June 14, 2021 Corresponding author: Bum-Suk Lee

Department of Physical Medicine and Rehabilitation, National Rehabilitation Center, 58 Samgaksan-ro, Gangbuk-gu, Seoul 01022, Korea. Tel: +82-2901-1886, Fax: +82-2-902-3835, E-mail: iambs@korea.kr

ORCID: EunYoung Kim (http://orcid.org/0000-0002-3122-7596); Hye Jin Lee (http://orcid.org/0000-0001-9400-6283); Onyoo Kim (http://orcid. org/0000-0001-9517-2240); In Suk Park (http://orcid.org/0000-0003-0627-3942); Bum-Suk Lee (http://orcid.org/0000-0002-0506-0403).

(c) This is an open-access article distributed under the terms of the Creative Commons Attribution Non-Commercial License (http://creativecommons.org/ licenses/by-nc/4.0) which permits unrestricted noncommercial use, distribution, and reproduction in any medium, provided the original work is properly cited. Copyright $\odot 2021$ by Korean Academy of Rehabilitation Medicine 


\section{INTRODUCTION}

The prevalence of urinary tract infection (UTI) is high in patients with spinal cord injury (SCI) [1]. Although mortality due to UTI has decreased from $43 \%$ in the 1940 s to $10 \%$ in the 1980 s and 1990 s [2], UTI remains a major cause of morbidity and mortality in patients with SCI [3]. Regular urodynamic study (UDS) is essential to reduce the morbidity and mortality due to UTI in patients with SCI. It is recommended to perform UDS every 3 months during the first year after injury, then at 1-year intervals thereafter [4]. While UDS is useful to identify abnormalities in the lower urinary tract, it is an invasive procedure and hence causes UTI and bacteriuria post-procedure [5]. Despite its invasiveness, every patient with SCI should still undergo UDS periodically. However, because the prevalence of post-UDS UTI has been reported to be as high as $\mathbf{8 . 6 \%}$, even in cases where sterile urine is confirmed [6], UDS is frequently delayed or canceled to prevent post-UDS UTI if asymptomatic bacteriuria or pyuria is found before the examination. This makes it difficult to reliably perform the procedure. Regarding safety, whether it is acceptable to perform the UDS when there is preUDS asymptomatic bacteriuria or pyuria in SCI patients is debatable because of these concerns. This is important, as it takes approximately 4-7 days to receive the results of urine culture. It is therefore more expeditious and useful to confirm the presence of pyuria using urinalysis, the result of which may determine whether UDS could be carried out safely. In the case of sterile pyuria, i.e., pyuria without bacteriuria, it is difficult to proceed with the test, as it is not thought to be safe. Nevertheless, there are only a few studies on the incidence of post-UDS UTI based on the presence of pyuria on urinalysis.

Therefore, this study aimed to investigate the difference in the incidence of post-UDS UTI between patients with and without pyuria on pre-UDS urinalysis, the result of which can be confirmed easily and rapidly in clinical circumstances. We also aimed to determine whether the use of prophylactic antibiotics for 5 days after UDS is beneficial for preventing UTI including cases of pre-UDS pyuria.

\section{MATERIALS AND METHODS}

\section{Study setting and population}

This is a retrospective study of all adult patients with
SCI who were admitted to our hospital from August 2015 to December 2018 and underwent UDS. Patients who had inaccurate or unknown nursing records before and after UDS, did not receive all standard tests, or had pre-UDS lower urinary tract symptoms (LUTS) such as fever, chilling, and dysuria, were excluded. Accordingly, UDS was conducted only if urinalysis performed before the examination showed sterile results, asymptomatic pyuria, or asymptomatic bacteriuria.

At the time of UDS, data of sex, age, diagnosis, injury type, neurologic level of injury (NLI), onset, duration, etiology, tetraplegia/paraplegia, underlying disease, and medication history were recorded for all patients.

Every patient received prophylactic antibiotics for 5 days beginning on the morning of the UDS regardless of the urinalysis and urine culture results. The antibiotics were ciprofloxacin $250 \mathrm{mg}$ twice daily, cefaclor $250 \mathrm{mg}$ three times daily, or trimethoprim/sulfamethoxazole (TMP/SMT) 400/80 mg twice daily.

Urinalysis and urine culture were performed in the morning and UDS was carried out in the afternoon. Because it takes 4-7 days to confirm culture results, only urinalysis results were available on the previous day of UDS. UDS was performed using the standard technique in a sterile setting. Urinalysis and urine culture were repeated 24 hours after UDS. LUTS, which includes, fever, chills, increasing spasticity, dysuria, and leukocytosis, were recorded for up to 7 days after UDS [7].

All study procedures were approved by Institutional Review Board at the National Rehabilitation Center and Hospital (NRC-2019-03-009), and the informed consent was wavied. We certify that all applicable institutional and governmental regulations concerning the ethical use of human volunteers were followed during the course of this research.

\section{Data definitions}

Bacteriuria was defined as a colony count of $>10^{5}$ colony forming units $/ \mathrm{mL}$ [8]. Because the specimen used in urinalysis was centrifuged urine, pyuria was defined when $>5$ white blood cells per high-power field were identified by microscopy [8-10]. Post-UDS UTI was diagnosed if bacteriuria occurred within 1 week after UDS [6] along with accompanying symptoms including fever, increasing incontinence and spasticity, malaise, or cloudy urine [11]. 
Voiding methods were analyzed and categorized as follows: indwelling catheters, subdivided into suprapubic and transurethral; intermittent catheterization; all other cases of urination without a catheter [12].

\section{Antibiotic-resistant bacteria}

Antibiotic resistance testing was performed on preand post-UDS urine cultures. Resistance to ciprofloxacin, cephalosporin, and TMP/SMT was identified in each cultured bacterium. If sensitive, the result was defined as sensitive, and if resistant or intermediate, the results were defined as being resistant. Resistance to ciprofloxacin or TMP/SMT was based on the corresponding antibiotics, and that to cephalosporin was based on cefuroxime axetil.

\section{Statistical analysis}

Categorical data were presented as frequency (\%) and numerical data as mean \pm standard deviation. For general characteristics of groups with and without pre-UDS pyuria, categorical data, such as sex, neurological level, and voiding method, were analyzed as chi-square test, and numerical data such as mean age and mean time since injury were analyzed as t-test. For determining the association of the incidence of symptomatic UTI with pyuria and bacteriuria, Fisher exact test was performed because all variables were categorical data and the expected value of some cells of the contingency table was found to be less than 5. $\mathrm{p}<0.05$ was defined as statistically significant. For antibiotic resistance analysis, the chi-square test was performed in triplicate between two groups each among the three groups (ciprofloxacin, cefaclor, TMP/SMT), in which $\mathrm{p}<0.01$ was defined as statistically significant to adjust for inflated type I error from multiple comparisons. All statistical analyses were performed using SPSS statistical analysis software, version 20.0 (IBM SPSS, Armonk, NY, USA).

\section{RESULTS}

\section{Study population}

From a total of 426 patients, 11 patients were excluded

Table 1. General characteristics of study population

\begin{tabular}{|c|c|c|c|}
\hline \multirow{2}{*}{ Variable } & \multicolumn{2}{|c|}{ Pre-UDS } & \multirow{2}{*}{ p-value ${ }^{\text {a) }}$} \\
\hline & Pyuria $(n=209)$ & No pyuria $(n=190)$ & \\
\hline Sex & & & 0.047 \\
\hline Male & $139(66.5)$ & $144(75.8)$ & \\
\hline Female & $70(33.5)$ & $46(24.2)$ & \\
\hline Neurological level & & & 0.035 \\
\hline Tetraplegia & $113(54.1)$ & $82(43.2)$ & \\
\hline Paraplegia & $96(45.9)$ & $108(56.8)$ & \\
\hline AIS & & & 0.000 \\
\hline A & $74(35.4)$ & $53(27.9)$ & \\
\hline B & $42(20.1)$ & $16(8.4)$ & \\
\hline $\mathrm{C}$ & $45(21.5)$ & $26(13.7)$ & \\
\hline $\mathrm{D}$ & $48(23.0)$ & $94(49.5)$ & \\
\hline $\mathrm{E}$ & $0(0)$ & $1(0.5)$ & \\
\hline Mean age (yr) & $49.9 \pm 17.1$ & $50.1 \pm 17.9$ & 0.492 \\
\hline Mean time since injury (mo) & $28.0 \pm 69.7$ & $21.0 \pm 57.7$ & 0.105 \\
\hline Voiding method & & & 0.000 \\
\hline Voiding without catheter & $36(17.2)$ & $88(46.3)$ & \\
\hline Intermittent catheterization & $93(44.5)$ & $89(46.8)$ & \\
\hline Transurethral indwelling catheter & $61(29.2)$ & $11(5.8)$ & \\
\hline Suprapubic indwelling catheter & $19(9.1)$ & $2(1.1)$ & \\
\hline
\end{tabular}

Values are presented as number of patients (\%).

UDS, urodynamic study; AIS, American Spinal cord Injury Association Impairment Scale.

${ }^{\text {a) }}$ Chi-square test for categorical data and t-test for numeric data. 
due to insufficient test records, 4 were excluded who missed pre-UDS culture, and 12 were of pre-adult age. A total of 399 patients with SCI (283 males and 116 females) were included in the final analysis. The general characteristics of groups with and without pre-UDS pyuria are summarized in Table 1.

\section{Association between the pyuria and bacteriuria}

Because patients with pre-UDS LUTS were excluded, all patients in the study were asymptomatic. Of the 399 patients, 209 patients $(52.4 \%)$ had pyuria on pre-UDS urinalysis, and 257 (64.4\%) had bacteriuria on pre-UDS culture (Table 2). In addition, 187 patients (46.9\%) had concurrently both bacteriuria and pyuria in pre-UDS urinalysis and urine culture; 22 patients (5.5\%) presented with pyuria only, 70 (17.5\%) bacteriuria only, and 120 $(32.6 \%)$ neither. Taken together, prediction of the presence of bacteriuria using the presence of pyuria had a sensitivity of $89.5 \%$ and a specificity of $63.2 \%$.

Association between the incidence of post-UDS UTI and the presence of pre-UDS pyuria or bacteriuria

Post-UDS UTI occurred in 6/399 (1.5\%) patients. In pre-

Table 2. Correlation between pre-UDS pyuria and preUDS bacteriuria $(\mathrm{n}=399)$

\begin{tabular}{lcrc}
\hline & Pyuria & No pyuria & Total \\
\hline Bacteriuria & $187(46.9)$ & $70(17.5)$ & $257(64.4)$ \\
No bacteriuria & $22(5.5)$ & $120(32.6)$ & $142(38.1)$ \\
Total & $209(52.4)$ & $190(48.1)$ & $399(100)$ \\
\hline
\end{tabular}

Values are presented as number of patients (\%).

UDS, urodynamic study.

Table 3. Symptomatic UTI incidence association with pyuria, bacteriuria $(\mathrm{n}=399)$

\begin{tabular}{llll}
\hline \multirow{2}{*}{ Subgroup } & \multicolumn{2}{c}{ Post-UDS } & \multirow{2}{*}{ p-value ${ }^{\text {a) }}$} \\
\cline { 2 - 3 } & No UTI & UTI & \\
\hline Pre-UDS urinalysis & & & \\
Pyuria (n=209) & $204(97.6)$ & $5(2.4)$ & 0.218 \\
\hline No pyuria (n=190) & $189(99.5)$ & $1(0.5)$ & \\
Pre-UDS urine culture & & & \\
Bacteriuria (n=257) & $253(98.4)$ & $4(1.6)$ & 1.000 \\
\hline No bacteriuria (n=142) & $140(98.6)$ & $2(1.4)$ & \\
\hline
\end{tabular}

Values are presented as number of patients (\%).

UTI, urinary tract infection; UDS, urodynamic study.

${ }^{a)}$ Fisher exact test.
UDS urinalysis, post-UDS UTI was observed in 5/209 (2.4\%) patients with pre-UDS pyuria and in $1 / 190(0.5 \%)$ patients without pre-UDS pyuria (Table 3 ). The difference between two groups was not statistically significant ( $\mathrm{p}=0.218)$. On pre-UDS culture, post-UDS UTI was found in $4 / 257$ (1.6\%) patients with bacteriuria and 2/142 (1.4\%) patients without bacteriuria. The difference between two groups was also not statistically significant $(\mathrm{p}=1.000)$.

\section{Characteristic of post-UDS UTI patients}

Six patients with post-UDS UTI comprised 5 patients with pyuria and 1 without pyuria on pre-UDS urinalysis. Of the 6 patients, 4 had bacteriuria and 2 did not on preUDS culture. This is shown in the summarized characteristics of the patients (Supplementary Table S1). Bacteria grown on cultures included Pseudomonas aeruginosa, Escherichia coli, Stenotrophomonas maltophilia, Acinetobacter lwoffii, and Klebsiella pneumoniae. All 6 patients complained of fever and received ciprofloxacin as antibiotic treatment. One patient presented with a bladder detrusor pressure higher than $40 \mathrm{~mL} / \mathrm{cmH}_{2} \mathrm{O}$. All 6 patients showed normal bladder capacity. A history of UTI within 1 month was recorded in one patient.

Risk factor evaluation was performed by comparing the 6 patients with post-UDS UTI (UTI group) to the other patients (no UTI group). No statistically significant differences in risk factors were found for the following variables: a history of UTI within 1 month, previous autonomic dysreflexia (AD), urinary incontinence, post-void residue, pyuria, bacteriuria, bladder emptying method, compliance, maximal detrusor pressure, or bladder capacity (Supplementary Table S2).

\section{Microbiology and prophylactic antibiotics}

Prophylactic antibiotics were administered for 399 patients, which were as follows: 291 (72.9\%), 75 (18.8\%),

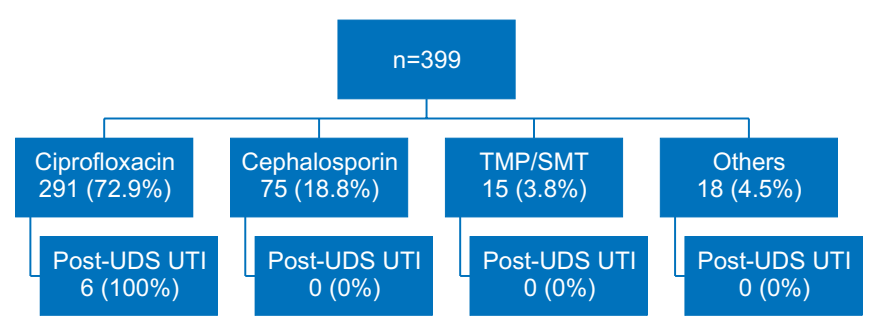

Fig. 1. Types of antibiotics prescribed before urodynamic study (UDS). UTI, urinary tract infection; TMP/SMT, trimethoprim/sulfamethoxazole. 
Table 4. Pre-UDS results of urine culture resistance for ciprofloxacin, cephalosporin and TMP/SMT

\begin{tabular}{lcccc}
\multicolumn{1}{c}{ Uropathogens } & Ciprofloxacin $^{\text {a) }}$ & Cephalosporin $^{\text {a) }}$ & TMP/SMT $^{\text {a) }}$ & Pre-UDS culture (n=230) \\
\hline Escherichia coli & $70 / 109(64.2)$ & $52 / 109(47.7)$ & $40 / 109(36.7)$ & $109(47.4)$ \\
Klebsiella pneumoniae & $24 / 48(50.0)$ & $23 / 48(47.9)$ & $19 / 48(39.6)$ & $48(20.9)$ \\
Pseudomonas aeruginosa & $4 / 12(33.3)$ & $12 / 12(100)$ & $12 / 12(100)$ & $12(5.2)$ \\
Proteus mirabilis & $9 / 12(75.0)$ & $8 / 12(66.7)$ & $9 / 12(75.0)$ & $12(5.2)$ \\
Others & $14 / 49(28.6)$ & $31 / 49(63.3)$ & $10 / 49(20.4)$ & $49(21.3)$ \\
Total & $121 / 230(52.6)$ & $126 / 230(54.8)$ & $90 / 230(39.1)$ & $230(100)$ \\
Gram-negative bacteria & $117 / 221(52.9)$ & $126 / 221(57.0)$ & $86 / 221(38.9)$ & $221(96.1)$ \\
\hline
\end{tabular}

Values are presented as number of patients (\%).

UDS, urodynamic study; TMP/SMT, trimethoprim/sulfamethoxazole; GNB, gram-negative bacteria.

${ }^{a)}$ Results of the number of resistance tests/total number of available tests.

$15(3.8 \%)$, and $18(4.5 \%)$ patients received ciprofloxacin, cephalosporin, TMP/SMT, and other antibiotics, respectively (Fig. 1). A total of 6 post-UDS UTI cases occurred from 291 patients prescribed ciprofloxacin.

Twenty-seven tests of 257 patients with pre-UDS bacteriuria showed non-specific results regarding antibiotic resistance and were excluded. Thus, regardless of the type of bacteria cultured, the results of antibiotic resistance testing for ciprofloxacin, cephalosporin, and TMP/SMT were analyzed in a total of 230 cases (Table 4). Among gram-negative strains (221 cases), resistance to ciprofloxacin, cephalosporin, and TMP/SMT were confirmed to be $52.9 \%$ (117 cases), $57.0 \%$ ( 126 cases), and $38.9 \%$ ( 86 cases), respectively. The results of intergroup chi-square test (Fig. 2) showed a significantly lower resistance to TMP/SMT than to ciprofloxacin $(\mathrm{p}<0.001)$; lower resistance to ciprofloxacin than to cephalosporin $(\mathrm{p}<0.001)$; and lower resistance to TMP/SMT than to cephalosporin ( $\mathrm{p}=0.001)$. E. coli (109 cases) accounted for the highest number of cultured strains, and the remainder included $K$. pneumonia, Enterococcus spp., $P$. aeruginosa, and Proteus mirabilis.

\section{DISCUSSION}

Because there are many SCI cases with asymptomatic pyuria and bacteriuria unrelated to UTI, clinicians often face a difficult choice on whether they should delay UDS in cases of pre-UDS pyuria or bacteriuria. In a retrospective study of 84 patients with SCI [13], 44.1\% showed asymptomatic pre-UDS bacteriuria. Likewise, in our study, pre-UDS pyuria and bacteriuria were found in 209/399 (52.4\%) and 257/399 (64.4\%) patients, respectively. If UDS



Fig. 2. Pre-urodynamic study results of urine culture resistance for ciprofloxacin, cephalosporin, and trimethoprim/sulfamethoxazole (TMP/SMT) in gram-negative isolates.

had been delayed in all patients with pyuria, more than a half of the total cases would have been deferred. Therefore, our study was undertaken to determine whether UDS could be performed as planned even in patients with SCI showing asymptomatic pyuria or bacteriuria.

Pre-UDS pyuria and bacteriuria are two predictive parameters for post-UDS UTI. Compared with bacteriuria, pyuria is easily clinically assessed, and can predict bacteriuria with a sensitivity of $89.5 \%$ and a specificity of $63.2 \%$, as seen in our study. This supports the findings of a similar prospective study of 56 patients with SCI, in which pyuria showed the highest sensitivity $(82.8 \%)$ and positive predictive value (93.8\%) for UTI [14]. Taken together, pyuria is clinically easy to detect and, due to its high predictive ability for bacteriuria and UTI, supports urinalysis for pre-UDS screening because it is an easier 
and more rapid procedure than is culture. Further, bacteriuria has several limitations as a marker for UTI in patients with SCI. Symptoms such as dysuria, which can be masked in patients with SCI due to sensory impairment in those with lower NLI affecting the urinary tract sensory function $[9,15]$, and urinary symptoms, such as urinary incontinence, can occur together, even in cases without UTI. In addition, it takes 4-7 days to confirm the result of culture. To date, studies regarding the association with post-UDS UTI have used bacteriuria as a parameter, and few studies have investigated the association of pyuria. A study of 133 patients with SCI showed that the incidence of post-UDS UTI in the pre-UDS sterile group and the pre-UDS bacteriuria group was $8.6 \%$ and $32.5 \%$, respectively, suggesting that pre-UDS bacteriuria is a risk factor for post-UDS UTI [6]. Conversely, another study of 72 patients with SCI demonstrated converse incidence of postUDS UTI in the pre-UDS sterile group and the pre-UDS bacteriuria group at $13.2 \%$ and $0 \%$, respectively, concluding that pre-UDS bacteriuria had a negligible effect on the occurrence of post-UDS UTI [16].

In our study, the prevalence of post-UDS UTI was investigated based on pre-UDS pyuria. Although 209 patients (52.4\%) had pyuria on pre-UDS analysis of 399 patients with asymptomatic SCI, no difference in the incidence of post-UDS UTI was significantly related to the presence of pre-UDS pyuria. The results of univariate analysis for risk factor evaluation suggested that pre-UDS pyuria was not a risk factor for post-UDS UTI. In a study analyzing risk factors for UDS in 140 patients with SCI [17], pyuria or bacteriuria was not found to be a risk factor for post-UDS UTI, which was consistent with the results of our study. However, since the incidence of UTI was much lower in our study than in other studies, there may be a possibility that no statistically significant result could be identified concerning risk factors for post-UDS UTI.

Although there is no consensus regarding the use of prophylactic antibiotics before UDS, it is recommended that prophylactic antibiotics should be used before all invasive procedures, including UDS in high-risk groups for UTI, similar to patients with SCI [9,18-22]. Conversely, a retrospective study of 661 patients with SCI [23] showed no significant difference in the incidence of post-UDS UTI between groups using and not using prophylactic antibiotics, and concluded that prophylactic antibiotics should not be used. Although an exact comparison is lim- ited by the study design, the incidence of post-UDS UTI in our study (1.5\%) was markedly lower than that in other studies $(9.7 \%-15.8 \%)[6,16]$. This may probably be due to prophylactic antibiotics used in the protocol. Moreover, the study not using prophylactic antibiotics showed a higher incidence rate than our study, even though UDS was performed only in cases that were sterile in pre-UDS analysis. Therefore, considering that patients with SCI are at a high risk for UTI, use of prophylactic antibiotics pre-UDS should be considered in this population.

In utilizing prophylactic antibiotics, the selection of appropriate antibiotics is important. According to an analysis of multidrug resistant (MDR) organisms from 2,629 culture samples collected between 2001 and 2013 [24], the incidence of MDR organism growth showed a gradually increasing trend, which was verified in another study [25]. In addition, MDR organisms were found more in patients with SCI than in the general population $[26,27]$ and were associated with increased mortality and poor outcomes in the long-term care of patients with SCI [28]. Hence, it is important to choose antibiotics that do not lead to MDR organism growth in patients with SCI; nevertheless, there is no consensus on the ideal antibiotic selection. It is difficult to decide first-line antibiotic choices because bacterial susceptibility patterns differ depending on geographical factors, although fluoroquinolone resistance has been found to increase regardless of geographic factors in several studies [25]. In the 230 cases with preUDS bacteriuria observed in our study, the resistance to TMP/SMT was $38.9 \%$, which was lower than that of both ciprofloxacin and cephalosporin, with statistically significant differences. Although TMP/SMT has a high tissue concentration and shows a therapeutic effect similar to ciprofloxacin, its use decreased due to growing resistance in Korea which started around the 2000s. However, bacterial resistance to TMP/SMT has recently declined in Korea, which might result from the long period of decreased use [29]. This result agrees with the result of a 6-year research on antibiotic resistance that noted a decreased resistance to antibiotics as the prescription frequency of the antibiotics decreased [30]. Considering the results of our study, the therapeutic effect of TMP/SMT on UTI, and a decreased resistance to TMP/SMT in Korea, TMP/ SMT may be considered for first-line antibiotic treatment in Korean patients with SCI.

Our study has three advantages. First, unlike other 
studies, pyuria was used as an associated parameter in the incidence of post-UDS UTI. Second, considering that aseptic procedures by an examiner are most important to prevent post-UDS UTI, this was an exceptional study that minimized potential confounding factors, possibly due to different procedure protocols in different centers, by including a large number of patients with SCI ( $n=399)$ from a single center using an identical protocol. Finally, this study included only hospitalized patients, which made it possible to scrutinize the signs, symptoms, and course related to the occurrence of post-UDS UTI for up to 7 days after the procedure.

Nevertheless, there are some limitations in this study. The number of patients with UTI was too small $(n=6)$ for risk factor evaluation. Because UDS was performed before release of the results of pre-UDS culture, ciprofloxacin, cefaclor or TMP/SMT were used as prophylactic antibiotics regardless of the results in all cases. In addition, because this was not a therapeutic comparative study, analysis of the type and duration of prophylactic antibiotic therapy was not performed. Further studies comparing the duration, type, and dose of antibiotic administration are needed to recommend accurate guidelines.

In conclusion, UDS is an essential examination for patients with SCI although it is an invasive procedure. No difference in the incidence of post-UDS UTI was observed related to the presence of pre-UDS pyuria. Therefore, when UDS is scheduled in patients with SCI, there is no need to delay the procedure, even in cases of pre-UDS pyuria. In addition, TMP/SMT may be considered for first-line antibiotics to prevent post-UDS UTI in Korea.

\section{CONFLICT OF INTEREST}

No potential conflict of interest relevant to this article was reported.

\section{AUTHOR CONTRIBUTION}

Conceptualization: Lee BS. Methodology: Lee BS. Formal analysis: Lee HJ, Kim OY, Park IS, Kim EY. Project administration: Kim EY, Park IS. Visualization: Kim EY. Writing-original draft: Kim EY, Lee BS. Writing-review and editing: Kim EY, Lee BS. Approval of final manuscript: all authors.

\section{SUPPLEMENTARY MATERIALS}

Supplementary materials can be found via http://doi. org/10.5535/arm.20241.

\section{REFERENCES}

1. Albayrak I, Caliskan A Levendoglu F, Ozerbil OM, Dagi HT. Characteristics of urinary tract infections in patients with spinal cord injuries hospitalized at a rehabilitation centre. Cyprus J Med Sci 2016;1:17-21.

2. Whiteneck GG, Charlifue SW, Frankel HL, Fraser MH, Gardner BP, Gerhart KA, et al. Mortality, morbidity, and psychosocial outcomes of persons spinal cord injured more than 20 years ago. Paraplegia 1992;30:61730.

3. Edokpolo LU, Stavris KB, Foster HE Jr. Intermittent catheterization and recurrent urinary tract infection in spinal cord injury. Top Spinal Cord Inj Rehabil 2012;18:187-92.

4. Khanna R, Sandhu AS, Doddamani D. Urodynamic management of neurogenic bladder in spinal cord injury. Med J Armed Forces India 2009;65:300-4.

5. Tsai SW, Kung FT, Chuang FC, Ou YC, Wu CJ, Huang $\mathrm{KH}$. Evaluation of the relationship between urodynamic examination and urinary tract infection based on urinalysis results. Taiwan J Obstet Gynecol 2013;52:493-7.

6. Bothig R, Fiebag K, Thietje R, Faschingbauer M, Hirschfeld S. Morbidity of urinary tract infection after urodynamic examination of hospitalized SCI patients: the impact of bladder management. Spinal Cord 2013;51:70-3.

7. Yang SO. Catheter-associated urinary tract infection. Korean J Urogenit Tract Infect Inflamm 2013;8:73-82.

8. Jameson JL, Fauci AS, Kasper DL, Hauser SL, Longo DL, Loscalzo L, editors. Harrison's principles of internal medicine. 20th ed. New York, NY: McGraw-Hill; 2018.

9. Rahimkhani M, Nikfallah A, Saberian M, Mordadi A, Varmazyar S, Tavakoli A. Urinary tract infection in spinal cord injuries. Asian J Phama Clin Res 2014;7:17882.

10. Tambyah PA, Maki DG. The relationship between pyuria and infection in patients with indwelling urinary 
catheters: a prospective study of 761 patients. Arch Intern Med 2000;160:673-7.

11. Goetz LL, Cardenas DD, Kennelly M, Bonne Lee BS, Linsenmeyer T, Moser C, et al. International spinal cord injury urinary tract infection basic data set. Spinal Cord 2013;51:700-4.

12. Oh SW, Jung JH, Cho IK, Lee HJ, Kwon SH, Lee BS. Changes in the trend in bladder emptying methods in patients with spinal cord injury: a 20-year single-center retrospective study. Ann Rehabil Med 2020;44:228-37.

13. Amaral DM, Pereira AM, Rodrigues MR, Gandarez MF, Cunha MR, Torres MS. Urinary tract infection in patients with spinal cord injury after urodynamics under fosfomycin prophylaxis: a retrospective analysis. Porto Biomed J 2019;4:e56.

14. Massa LM, Hoffman JM, Cardenas DD. Validity, accuracy, and predictive value of urinary tract infection signs and symptoms in individuals with spinal cord injury on intermittent catheterization. J Spinal Cord Med 2009;32:568-73.

15. Pannek J. Treatment of urinary tract infection in persons with spinal cord injury: guidelines, evidence, and clinical practice: a questionnaire-based survey and review of the literature. J Spinal Cord Med 2011;34:11-5.

16. Pannek J, Nehiba M. Morbidity of urodynamic testing in patients with spinal cord injury: is antibiotic prophylaxis necessary? Spinal Cord 2007;45:771-4.

17. Hwang SI, Lee BS, Han ZA, Lee HJ, Han SH, Kim MO. Factors related to the occurrence of urinary tract infection following a urodynamic study in patients with spinal cord injury. Ann Rehabil Med 2016;40:718-24.

18. Cameron AP, Campeau L, Brucker BM, Clemens JQ, Bales GT, Albo ME, et al. Best practice policy statement on urodynamic antibiotic prophylaxis in the non-index patient. Neurourol Urodyn 2017;36:915-26.

19. Litza JA, Brill JR. Urinary tract infections. Prim Care 2010;37:491-507.

20. Wagenlehner FM, Wagenlehner C, Schinzel S, Naber KG; Working Group "Urological Infections" of German Society of Urology. Prospective, randomized, multicentric, open, comparative study on the efficacy of a prophylactic single dose of $500 \mathrm{mg}$ levofloxacin versus $1920 \mathrm{mg}$ trimethoprim/sulfamethoxazole versus a control group in patients undergoing TUR of the prostate. Eur Urol 2005;47:549-56.

21. Hirakauva EY, Bianchi-Ferraro AM, Zucchi EV, Kaji- kawa MM, Girao MJ, Sartori MG, et al. Incidence of Bacteriuria after Urodynamic Study with or without Antibiotic Prophylaxis in Women with Urinary Incontinence. Rev Bras Ginecol Obstet 2017;39:534-40.

22. Ditunno JF, Donovan WH, Oleson CV. History of spinal cord medicine. In: Kirshblum S, Lin VW, editors. Spinal cord medicine. 3rd ed. New York, NY: Demos Medical; 2018. pp. 2-10.

23. da Silva MT, Barboza AL, Pijoan MM, Beraldo PS. Antibiotic prophylaxis prior to urodynamic study in patients with traumatic spinal cord injury: is there an indication? Int Braz J Urol 2019;45:347-53.

24. Kang MS, Lee BS, Lee HJ, Hwang SW, Han ZA. Prevalence of and risk factors for multidrug-resistant bacteria in urine cultures of spinal cord injury patients. Ann Rehabil Med 2015;39:686-95.

25. Fitzpatrick MA, Suda KJ, Safdar N, Burns SP, Jones MM, Poggensee L, et al. Changes in bacterial epidemiology and antibiotic resistance among veterans with spinal cord injury/disorder over the past 9 years. J Spinal Cord Med 2018;41:199-207.

26. Suda KJ, Patel UC, Sabzwari R, Cao L, Ramanathan S, Hill JN, et al. Bacterial susceptibility patterns in patients with spinal cord injury and disorder (SCI/D): an opportunity for customized stewardship tools. Spinal Cord 2016;54:1001-9.

27. Yoon SB, Lee BS, Lee KD, Hwang SI, Lee HJ, Han ZA. Comparison of bacterial strains and antibiotic susceptibilities in urinary isolates of spinal cord injury patients from the community and hospital. Spinal Cord 2014;52:298-301.

28. Ramanathan S, Fitzpatrick MA, Suda KJ, Burns SP, Jones MM, LaVela et al. Multidrug-resistant gramnegative organisms and association with 1-year mortality, readmission, and length of stay in Veterans with spinal cord injuries and disorders. Spinal Cord 2020;58:596-608.

29. Kim HY, Lee SJ, Lee DS, Yoo JM, Choe HS. Microbiological characteristics of unresolved acute uncomplicated cystitis. Microb Drug Resist 2016;22:387-91.

30. Kitagawa K, Shigemura K, Nomi M, Takami N, Yamada N, Fujisawa $M$. Use of oral third generation cephalosporins and quinolones and occurrence of antibiotic-resistant strains in the neurogenic bladder (NB) outpatient setting: a retrospective chart audit. Spinal Cord 2020;58:705-10. 